CfPA-TH-95-15

astro-ph/9508040

2018 April 12

\title{
THE FIRST GENERATION OF STARS: FIRST STEPS TOWARDS CHEMICAL EVOLUTION OF GALAXIES
}

\author{
Jean Audouze \\ Institut d'Astrophysique du CNRS \\ 98 bis Boulevard Arago, 75014 Paris, France \\ and Joseph Silk \\ Center for Particle Astrophysics \\ and Department of Astronomy, \\ University of California, Berkeley, CA 94720-7304
}

\begin{abstract}
We argue that extreme metal-poor stars show a high dispersion in metallicity, because their abundances are the outcome of very few supernova events. Abundance anomalies should appear because of the discrete range of progenitor masses. There is a natural metallicity threshold of $Z / Z_{\odot} \sim 10^{-4}$ below which one would expect to find very few, if any, halo stars. Similar reasoning is applied to lower mass systems, such as metal-poor compact blue galaxies and Lyman alpha absorption line clouds seen towards high redshift quasars, where a somewhat higher threshold is inferred.
\end{abstract}

Subject headings: Galaxy: formation-Galaxy: halo-stars:abundances 


\section{Introduction}

A recent analysis (McWilliam, Preston, Sneden and Searle 1995) of extremely metalpoor stars to $[\mathrm{Fe} / \mathrm{H}] \gtrsim-4$, found remarkable trends concerning many abundances, including those of alpha-elements $(\mathrm{Mg}, \mathrm{Si}, \mathrm{Ca})$, iron peak elements ( $\mathrm{Sc}, \mathrm{Ti}, \mathrm{Cr}, \mathrm{Mn}, \mathrm{Co}, \mathrm{Ni}$ ) and heavy elements $(\mathrm{Y}, \mathrm{Sr}, \mathrm{Ba}, \mathrm{Eu})$, with respect to $[\mathrm{Fe} / \mathrm{H}]$, that appear over the range $[\mathrm{Fe} / \mathrm{H}]=-4$ to -2.4 . A large abundance dispersion was also found between different stars, for the most metal-poor stars $([\mathrm{Fe} / \mathrm{H}] \gtrsim-4)$. We shall argue that these data provide novel clues about the nucleosynthetic role of the first generation of stars. We interpret these variations and trends as the signature of an epoch of galactic evolution before mixing of stellar ejecta had time to be efficient. This constraint sets a metallicity threshold, we shall show, of $[\mathrm{Fe} / \mathrm{H}] \approx-4$. We also discuss similar abundance trends observed in blue compact galaxies and high redshift metal-poor quasar absorption line systems.

\section{The first generation of galactic stars}

A general timescale argument for the early enrichment is as follows: the star formation rate in the disk of our galaxy is constant, to within a factor of 2, over the past 10 Gyr. In a simple closed box model, one would expect, over the initial lifetime of a single generation of massive stars of approximately $2 \times 10^{6} \mathrm{yr}$, to attain a metallicity of roughly $2 \times 10^{-4}$ of the solar value. This implies that only one generation of massive stars had time to explode as Type II supernovae and eject metal-enriched material into the interstellar medium from which these extremely metal-poor stars were formed.

An estimate of the first metal enrichment is as follows. The number of Type II supernovae that would have exploded in this initial period should be $N \sim 1-2 \times 10^{4}$, if their rate is comparable to the present rate of Type II supernovae, namely 1 per $100 \mathrm{yr}$. This should be a conservative estimate, since considerations of the diminished role of mass loss for zero-metal stars (Jura 1986) and of prompt initial enrichment (Truran and Cameron 1971) suggest that the rate of Type II supernovae may have been higher in the early galaxy. Assuming that each Type II supernova ejects approximately $1 M_{\odot}$ of metals, we infer a mean initial enrichment of $Z=3 \times 10^{-6} M_{10}$, where the mass in Population II is written as $10^{10} M_{10} M_{\odot}$.

However we note that the interstellar medium enrichment will be highly inhomogeneous during this early phase of the galaxy. The reason for this is that the mean separation between supernova remnants is $(V / N)^{1 / 3}$, where $V$ is the volume of the spheroid, $\sim 10^{11} \mathrm{pc}^{3}$, or about 100 to $200 \mathrm{pc}$. The maximum extent of a supernova remnant before breakup and dispersion into the interstellar medium should be about half the mean separation. We may utilize theoretical calculations of the evolution of radiative supernova remnants evolving in a homogeneous interstellar medium (Cioffi, McKee and Bertschinger 1988) to estimate a mean size when the remnant finally merges with the ambient medium of $69\left[E_{51}^{0.32} n^{-0.37} \sigma_{10}^{-0.43} \zeta^{0.09}\right] \mathrm{pc}$. Here $E_{51}$ is the initial kinetic energy of the supernova remnant in units of $10^{51} \mathrm{ergs}, n$ is the ambient density of the interstellar medium in particles $\mathrm{cm}^{-3}, \sigma_{10}$ is the velocity dispersion in units of $10 \mathrm{~km} \mathrm{~s}^{-1}$ of the interstellar gas that provides the effective pressure, and $\zeta$ is the ISM metallicity, relative to the solar value. The merger time for a remnant is $2 \times 10^{6}\left[E_{51}^{0.32} n^{-0.47} \sigma_{10}^{-1.43} \zeta^{-0.05}\right]$ yr. To this we add the main sequence lifetime of $\mathrm{a} \sim 20 \mathrm{M}_{\odot}$ star to infer a total mixing time for the first generation of stars of about $3 \times 10^{6} \mathrm{yr}$. The typical volume element of interstellar gas will be exposed, during this initial period of marginal mixing, to at most 2-3 supernova remnants. The predecessor stars will not be of precisely the same mass, but will necessarily span the range $10-100 \mathrm{M}_{\odot}$. Abundance calculations for metal-poor massive stars show that the nucleosynthetic yields are sensitive to the progenitor mass (Woosley and Weaver 1995). We 
deduce that chemical inhomogeneities will be maximal until the number of SN remnants has increased by 1 or 2 orders of magnitude. This spans the range in the metallicities of the oldest stars from $[\mathrm{Fe} / \mathrm{H}]=-4$ to $\sim-2.5$, as noted by McWilliam et al. (1995).

Among the many abundances determined by McWilliam et al. (1995), several deserve further consideration. Having provided an explanation for the star-to-star dispersion at low metallicity, we note the following abundance trends regarding two classes of elements, the alpha elements ranging from sodium to calcium and the iron peak elements including scandium, titanium, chromium, manganese, cobalt and nickel. Relative to iron, the stars with metallicity below $[\mathrm{Fe} / \mathrm{H}]=-2.4$ are on average relatively rich in magnesium, calcium and cobalt, but are relatively low in aluminium, chromium, manganese and nickel. For other elements such as sodium, scandium and titanium, the dispersion in abundances is large. For the alpha elements, the trends are consistent with what is expected, namely an overabundance of even-A nuclei and relative underabundance of odd-A elements because of the low neutronization relative to the alpha flux in very metal-poor material. The situation is not the same for Fe peak nuclei, for which the relative abundances depend on the mass cut of the supernova progenitor, via the temperature of the stellar material which induces the specific photodisintegration processes (Woosley and Weaver 1986). For most of the stars considered, the s-process element abundances are weak, as is consistent with the relative lack of available neutron flux. Nevertheless, there are conspicuous exceptions, especially for the star CS 22898-027 with $[\mathrm{Fe} / \mathrm{H}]=-2.35$, where barium and other sprocess elements are overabundant with respect to iron by factors of about 300 . It is worth noting that this specific star has a metallicity 30 times as high as the most metalpoor stars, and therefore there would have been time for pre-enrichment in elements such as ${ }^{22} \mathrm{Ne}$, the likely neutron source. This is consistent with the interpretation of this star as a CH subgiant (Thorburn and Beers 1992), for which mass exchange may account for enhanced s-process elemental abundances.

We now focus attention on the three stars with the lowest $\left(Z / Z_{\odot} \approx 10^{-4}\right)$ abundances, analyzed by McWilliam et al. (1995), namely CS 22885-096 ([Fe/H] = -3.79); CS 22949$037([\mathrm{Fe} / \mathrm{H}]=-3.99) ; \mathrm{CD}-38245([\mathrm{Fe} / \mathrm{H}]=-4.01)$. All three stars are relatively rich in magnesium, calcium, scandium and cobalt relative to iron, but are relatively poor in chromium and manganese. Nickel, aluminium and titanium track iron, as do barium and strontium. The alpha- and heavy element abundances behave as expected for massive low metallicity stars. As anticipated (Table 1), iron peak elements from scandium to nickel show a wide range of abundances in the computations of Woosley and Weaver (1995), depending on both the progenitor mass and on the mass cut. These are the only calculations we have found that study zero-metal massive star explosions. In addition to uncertainties in the final stages of stellar evolution, the mass cut is questionable because the role of winds from evolved low metallicity stars is poorly known. It seems likely that a range of masses and mass cuts will readily account for the abundance variations found for iron peak elements in the extremely low metallicity stars. We must add together the yields of at least 2 , and probably no more than 3, distinct mass models, given the average supernova remnant separation and the total mixing time for the first generation of stars. As an illustration of this, we note that the observed elemental abundance patterns (displayed in Table 1) are reasonably consistent with the variations in the mass models and mass cuts, for example, if we combine the yields from 13 and $22 \mathrm{M}_{\odot}$ stars (models Z13A and Z22A, respectively) with initially zero metallicity. Model Z13A is in agreement with the abundance pattern of $\mathrm{Al}, \mathrm{Cr}, \mathrm{Mn}, \mathrm{Co}$, and $\mathrm{Ni}$ in CS22885-096, whereas the abundances of $\mathrm{Mg}, \mathrm{Si}, \mathrm{Ca}, \mathrm{Sc}$ and $\mathrm{Ni}$ in CD-38 245 are in better accord with model Z22A. One should note that these estimates fail to satisfactorily reproduce the observed $\mathrm{Co} / \mathrm{Fe}$ overabundance. However these observations are unexpected given that Co is an odd iron peak element. 
Once there has been time for many generations (e.g. 10) of massive stars, we expect that these variations would mostly be suppressed in the integrated contributions from a range of stellar masses. In addition, we note that the three extreme metal-poor stars show the same spread in strontium and barium abundances as do the more metal-rich stars. This means that some of the metal-poor progenitor stars should have undergone mixing of carbon-rich, helium-rich and hydrogen-rich layers in order to produce $\mathrm{N}$ by the $\mathrm{CN}$ cycle, followed by the alpha particle-induced reactions ${ }^{14} N(\alpha, \gamma){ }^{18} F\left(\beta^{+}\right){ }^{18} O(\alpha, \gamma){ }^{22} N e$.

It is worth mentioning that our interpretation of the data on extremely metal-poor stars is subject to a direct observational test. If the stars with $[\mathrm{Fe} / \mathrm{H}] \approx 10^{-4}$ are the outcome of the first generation of massive stars and their associated supernovae, one would not expect to find any stars with appreciably lower metallicity. This is because the $\sim 10^{4}$ supernovae will have already produced sufficient enrichment so that very little gas would have retained its primordial abundances. It is worth noting in this respect that Norris, Peterson and Beers (1993) and McWilliam et al. (1995), despite intensive searches, find their lowest metallicity candidate stars to be just near the $[\mathrm{Fe} / \mathrm{H}]=-4$ limit. Our conclusion can be compared with that of Beers (1995), who uses an iron enrichment rate per supernova adopted by Primas, Molaro and Castelli (1994) that is significantly lower than our preferred value that we have taken from the Woosley-Weaver $(1986,1985)$ prescriptions. With the lower yield, Beers infers that we should observe a few stars with $[\mathrm{Fe} / \mathrm{H}] \approx-5$ in the next few years. While we would advocate a higher threshold, we can argue that in any circumstances the observed lower limit on metallicity in the extremely metal-poor halo stars should provide a direct estimate of the average supernova metal enrichment rate.

\section{Metal-poor extragalactic systems}

Analogous reasoning can be applied to metal-poor extragalactic systems, including blue compact galaxies and Lyman alpha absorption line clouds seen along the line of sight to quasars. Blue compact galaxies are vigorously forming stars at the present epoch, at a rate such that the observed level of metal enrichment implies that we may be witnessing their first major episode of star formation and associated chemical evolution. Consider the classic case of the most metal-poor blue compact galaxy known, IZw18. Kunth, Lequeux, Sargent and Viallefond (1994) have reanalyzed the oxygen and silicon abundances in the surrounding neutral gas region. Although the abundance determinations are uncertain, their best estimates are $O / H=7.9 \times 10^{-7}$ and $S i / H=7.5 \times 10^{-8}$, corresponding respectively to $10^{-3}$ and $2 \times 10^{-3}$ of the solar value. As noted by these authors, the composition of IZw18 should be close to the primordial value, and its helium abundance should therefore reflect the Big Bang nucleosynthesis prediction (to the extent that one can accurately estimate this abundance, which is still far from the case, see e.g. Pagel, Simonson, Terlevich and Edmunds 1992). The emission line region metallicity is about 1/30 of the solar value and reflects the current star burst phase, which has presumably been underway for $\sim 10^{8}$ yr. Hence any "primordial" gas outside the emission line region should have been contaminated only by the first generation of stars, that we assume occurred throughout IZw18. This would result in an abundance level equal to the ratio of the lifetime of the first stellar generation $\left(\sim 3 \times 10^{6}\right.$ yr $)$ to the age of the current star burst, which has produced the observed emission line gas metallicity of -1.5 . This results in a predicted metallicity for the gas outside the emission line region of about $10^{-3}$ of the solar value.

We point out that in the most metal-poor blue compact galaxy IZw18, the relative [Si/O] ratio determined by Kunth et al. (1994) is 0.3 (or 2 by number). This is in agreement with the fact that alpha particle elements with even $\mathrm{A}$ are produced more copiously than $\mathrm{O}$, and a fortiori Fe. Moreover the various compilations of He versus $\mathrm{O}$ and $\mathrm{N}$ clearly show 
a large dispersion in metallicity, comparable to those observed in low metal stars (see e.g. Pagel 1995).

A similar approach can be adopted for pregalactic clouds, such as those observed in absorption towards high redshift quasars, where low metallicities are measured. The most primitive clouds are those of the Lyman alpha forest, where recent work has shown that the metallicity is about $1 / 300$ of the solar value (Cowie 1995). These are believed to be low mass clouds comparable in mass to, and likely precursors of, dwarf galaxies, and the metallicity produced by the first generation of stars is again expected to be on the order of $10^{-3}$ of the solar value.

For damped Lyman alpha systems, often considered to be the precursors of disks, comparably low metallicities are also measured in some cases, but span a wide range. Recently two independent teams (Songaila, Cowie, Hogan and Rugers 1994; Carswell, Rauch, Weymann, Cooke and Webb 1994) have reported the determination of a very large D/H abundance ratio, $2.4-3.0 \times 10^{-4}$, in the direction of $\mathrm{Q} 0014+813$ at $z=3.32$, with the associated metallicity estimated to lie between -3.5 and -1.6 . Such a high D abundance, if confirmed, implies a low baryon density, still consistent with the visible matter density, at the cost, however, of producing an excessive amount of ${ }^{3} \mathrm{He}$ (see, e.g. Palla, Galli and Silk, 1995; Vangioni-Flam and Cassé 1995). If the lower metallicity determination is correct, there has only been enrichment by a first generation of stars, implying a negligible level of astration.

However Tytler (1995) has reported detection of D at high redshift in another line of sight at a level that is lower by an order of magnitude. If astration were responsible for the low $\mathrm{D} / \mathrm{H}$ value in this component, there would have been several generations of massive stars, and the metal abundance would be correspondingly enhanced. It has been recently argued that in a highly inhomogeneous early universe dominated by primordial entropy fluctuations on scales up to those of a galactic mass, primordial nucleosynthesis may result in large line-of-sight variations in the primordial D/H ratio (Jedamzik, Mathews and Fuller 1995), at the price however of a high primordial ${ }^{7} \mathrm{Li} / \mathrm{H}$ ratio and relatively low ${ }^{4} \mathrm{He}$ mass fraction. In this situation, primordial conditions rather than astration are responsible for the variations in $\mathrm{D} / \mathrm{H}$. Given the importance of such an inference, further determinations of both metallicity and deuterium abundances in similar clouds at high redshift are of considerable importance.

\section{Conclusion}

Recent observations of extremely metal-poor halo stars provide unique clues about the first steps of the chemical evolution history of our galaxy. We interpret the large dispersion in elemental abundances as a manifestation of an early phase of enrichment when there were only a few supernova sources and mixing of their ejecta was incomplete. A consequence of this interpretation is that we predict the absence of any halo stars of appreciably lower metallicity. A similar interpretation has previously been applied to account for the absence of emission line dwarf galaxies with abundances below those of the metal-poor compact blue galaxy IZw18 (Kunth and Sargent 1986). We have extended this argument to account for the observed abundance differences between Si and O (Kunth et al. 1994). Moreover the dispersion in abundances between different blue compact galaxies is a consequence of the earliest phase of chemical evolution associated with the first generations of massive stars. We have also applied a similar argument to Lyman alpha absorption line systems at high redshift, where we may be witnessing gas and deuterium-rich precursors to dwarf and to disk galaxies, corresponding to Lyman alpha forest and damped Lyman alpha systems, respectively. 


\section{Acknowledgements}

This research has been supported in part at the University of California, Berkeley by the NSF through the Center for Particle Astrophysics, and in part by a grant from the D.O.E.; J. A. also wishes to gratefully acknowledge a stimulating conversation with N. Prantzos, support as a Regents' Lecturer at the University of California, Berkeley, and the hospitality of the Berkeley Department of Astronomy and the Center for Particle Astrophysics. We also thank T. Beers and J. Truran for useful comments.

\section{References}

Anders, E., and Grevesse, N. 1989, Geochim. Cosmochim. Acta, 53, 197.

Beers, T. C. 1995, in The Light Element Abundances, ed. P. Crane (New York: Springer), 145.

Carswell, R.F., Rauch, M., Weymann, R.J., Cooke, A.J., and Webb, J.K. 1994 Mon. Not. R. astr. Soc., 268, L1.

Cioffi, D. F., McKee, C. F. and Bertschinger, E. 1988, ApJ, 334, 252.

Cowie, L. L. 1995, preprint.

Jedamzik, K., Mathews, G. J. and Fuller, G. M. 1995, ApJ, 441, 465.

Jura, M. 1986, ApJ,301, 624.

Kunth, D., Lequeux, J., Sargent, W. L. W. and Viallefond, F. 1994, Astr. Ap., 282, 709.

Kunth, D. and Sargent, W. L. W. 1986, ApJ, 300, 496.

McWilliam, A., Preston, G., Sneden, C. and Searle, L. 1995, A. J. (in press).

Norris, J. E., Peterson, R. C. and Beers, T. C. 1993, ApJ, 415, 797.

Pagel, B. E. J. 1995, IAC Winter School on Galaxy Formation and Evolution, ed. C. Muñoz-Turón (Cambridge University Press, in press).

Pagel, B. E. J., Simonson, E. A., Terlevich, R. J. and Edmunds, M. G. 1992, M.N.R.A.S., 255,325 .

Palla, F., Galli, D. and Silk, J. 1995, ApJ (in press).

Songaila, A., Cowie, L.L., Hogan, C.J., and Rugers, M. 1994, Nature, 368, 599.

Primas, F., Molaro, P. and Castelli, F. 1994, Astr. .Ap., 290, 885.

Thorburn and Beers, T. 1992, BAAS, 24, 1278.

Truran, J. W. and Cameron, A. G. W. 1971, Astr. Space Sci., 14, 179.

Tytler, D., 1995, ESO Meeting on Quasar Absorption lines, in press

Vangioni-Flam, E. and Cassé, M. 1995, ApJ, 441, 471.

Woosley, S. E. and Weaver, T. A. 1986, Ann. rev. Astr. Ap., 24, 205.

Woosley, S. E. and Weaver, T. A. 1995, ApJSuppl, in press. 
TABLE 1

Comparison of Abundances in 3 Extreme Metal-Poor Stars with Supernova Models ${ }^{1}$

\begin{tabular}{cccccc}
\hline \hline Element & ${ }^{2} \mathrm{CD}-38245$ & ${ }^{2} \mathrm{CS} 22949-037$ & ${ }^{2} \mathrm{CS} 22885-096$ & ${ }^{3} \mathrm{Z} 13 \mathrm{~A}$ & ${ }^{3} \mathrm{Z} 22 \mathrm{~A}$ \\
{$[\mathrm{Fe} / \mathrm{H}]$} & -4.01 & -3.99 & -3.79 & - & - \\
$\mathrm{C}$ & -0.1 & 0.88 & 0.3 & -0.87 & -0.20 \\
$\mathrm{Na}$ & -0.1 & 2.16 & -0.11 & -1.46 & -0.09 \\
$\mathrm{Mg}$ & 0.38 & 1.20 & 0.46 & -0.77 & 0.10 \\
$\mathrm{Al}$ & -0.99 & -0.07 & -0.80 & -1.24 & -0.53 \\
$\mathrm{Si}$ & 0.66 & 0.86 & 0.69 & -0.50 & 0.24 \\
$\mathrm{Ca}$ & 0.58 & 0.90 & 0.58 & -0.52 & 0.44 \\
$\mathrm{Sc}$ & 0.18 & 0.86 & 0.47 & -0.61 & 0.03 \\
$\mathrm{Ti}$ & 0.79 & 0.53 & 1.78 & -0.33 & -0.02 \\
$\mathrm{Cr}$ & -0.50 & -0.49 & -0.49 & -0.31 & 0.03 \\
$\mathrm{Mn}$ & -0.08 & & -0.78 & -0.67 & -0.44 \\
$\mathrm{Co}$ & 0.44 & 0.53 & 0.35 & 0.05 & -0.33 \\
$\mathrm{Ni}$ & -0.12 & & 0.83 & 0.35 & -0.10 \\
$\mathrm{Sr}$ & -0.61 & 0.42 & -135 & & \\
$\mathrm{Ba}$ & 0.02 & -0.16 & & & \\
\hline
\end{tabular}

1 The observed stellar abundances and the model abundances, expressed with respect to the tabulation by Anders and Grevesse (1989), are given logarithmically with respect to the iron abundance.

${ }^{2}$ Abundances from McWilliam et al.(1995).

${ }^{3}$ Metal-free supernova models from Woosley and Weaver (1995): models Z13A and Z22A have initial masses respectively of 13 and $22 \mathrm{M}_{\odot}$. 